\title{
Higher-Order Rytov Approximation for Large-Scale and Strong Perturbation Media
}

\author{
Bo Feng ${ }^{1, *}$, Ru-Shan $\mathrm{Wu}^{2}$ and Huazhong Wang ${ }^{1}$ \\ ${ }^{1}$ Wave phenomena and inversion imaging group (WPI), School of Ocean and Earth \\ Science, Tongji University, Shanghai, 200092, China. \\ ${ }^{2}$ Modeling and Imaging Laboratory, University of California, Santa Cruz, 95060, \\ CA, USA.
}

Received 4 April 2018; Accepted (in revised version) 24 October 2018

\begin{abstract}
In the field of geophysics, although the first-order Rytov approximation is widely used, the higher-order approximation is seldom discussed. From both theoretical analysis and numerical tests, the accumulated phase error introduced in the first-order Rytov approximation cannot be neglected in the presence of strong velocity perturbation. In this paper, we are focused on improving the phase accuracy of forward scattered wavefield, especially for the large-scale and strong velocity perturbation case. We develop an equivalent source method which can update the imaginary part of the complex phase iteratively, and the higher-order scattered wavefield can be approximated by multiplying the incident wavefield by the exponent of the imaginary part of the complex phase. Although the convergence of the proposed method has not been proved mathematically, numerical examples demonstrate that our method can produce an improved accuracy for traveltime (phase) prediction, even for strong perturbation media. However, due to the neglect of the real part of the complex phase, the amplitude change of the scattered wavefield cannot be recovered. Furthermore, in the presence of multi-arrivals phenomenon, the equivalent scattering source should be handled carefully due to the multi-directions of the wavefield. Further investigations should be done to improve the applicability of the proposed method.
\end{abstract}

AMS subject classifications: 74J20, 86A15

Key words: Rytov approximation, higher-order approximation, large-scale strong perturbation, forward scattering, equivalent scattering source.

\section{Introduction}

From the analysis of scattering characteristics, we know that the forescattering is controlled by the D.C. component (zero-wavenumber part and long wavelength component)

\footnotetext{
*Corresponding author. Email addresses: ancd111@163.com (B. Feng), rwu@ucsc.edu (R.-S. Wu),
} herbhuak@vip. 163.com (H. Wang) 
of the medium spectrum [1,2]. The D.C. component of the medium spectrum increases almost linearly with the propagation distance in general. The validity condition for the Born approximation is the smallness of the scattered field compared with the incident field. Therefore, in the case of long-range propagation of forward scattering, due to the accumulation of phase changes caused by the velocity perturbations, the validity of the Born approximation is easily to be violated. However, phase-change accumulation can be easily handled by the Rytov transformation. This is why the Rytov approximation has been widely used for long distance propagation with only forescattering or small-angle scattering involved, such as the line-of-sight propagation of optical or radio waves [3-5], transmission fluctuations of seismic waves at arrays [6-8], diffraction tomography [9-12], seismic imaging using one-way propagators [13] and the calculation of finite-frequency sensitivity kernel for travel-time tomography [14-17].

It is well-known that for long-range wave propagation in the regime of small-angle scattering, Rytov approximation is superior to the Born approximation. However, the accuracy and convergence of Rytov series is still not established. Some discussions on the validity conditions are in the literatures [4,18-22]. In the area of diffraction tomography, it is clear that the first-order Rytov approximation is not accurate enough for large strongcontrast media in forward and inverse problems, therefore higher-order Rytov inversion was proposed and the results showed improved inversion accuracy [23]. For statistical wave propagation problem, Manning [24] derived a second-order Rytov approximation for general beam wave propagation through turbulent media. Kim and Tinin [25] used the second-order Rytov approximation to calculate the ionospheric residual error of dualfrequency satellite navigation system, which considered the diffraction arising where the Fresnel radius becomes larger than the inner scale of the spectrum of ionospheric turbulence.

Different from previous researches, we mainly focus on increasing the phase accuracy of the scattered wavefield in order to develop a non-linear traveltime inversion method for strong-perturbation media. This paper is organized as following: In the second section, we will demonstrate how to iteratively approximate the imaginary part of the complex phase function. In the third section, we will use numerical examples to show the improvements of the proposed higher-order Rytov approximation method over the firstorder Rytov approximation. Finally the perspectives and limitations of our method are discussed.

\section{Method}

Let $u_{0}(\mathbf{x} ; \omega)$ be the solution in the absence of perturbation, i.e.,

$$
\left(\nabla^{2}+k^{2}\right) u_{0}=0
$$

where the wave-number $k$ is defined as: $k=\omega / c_{0}(\mathbf{x})$ and $c_{0}(\mathbf{x})$ is the background velocity, $u_{0}=u_{0}(\mathbf{x} ; \omega)$ is the unperturbed wave field. 
Suppose the perturbed wave field after interaction with the heterogeneity $\varepsilon(\mathbf{x})$ is $u(\mathbf{x} ; \omega)$, where $\varepsilon(\mathbf{x})=c_{0}^{2}(\mathbf{x}) / c^{2}(\mathbf{x})-1$, and $c(\mathbf{x})$ is the velocity model containing the heterogeneity. We normalize $u(\mathbf{x} ; \omega)$ by the unperturbed field $u_{0}(\mathbf{x} ; \omega)$ and express the perturbation of the field by a complex phase perturbation function $\psi(\mathbf{x} ; \omega)$, i.e.,

$$
u(\mathbf{x} ; \omega) / u_{0}(\mathbf{x} ; \omega)=e^{\psi(\mathbf{x} ; \omega)} .
$$

The accurate expression for the complex phase $[4,22,26]$ is

$$
\psi(\mathbf{x})=\frac{1}{u_{0}(\mathbf{x})} \int_{V}\left(k^{2} \varepsilon\left(\mathbf{x}^{\prime}\right)+\nabla \psi\left(\mathbf{x}^{\prime}\right) \cdot \nabla \psi\left(\mathbf{x}^{\prime}\right),\right) G_{0}\left(\mathbf{x} ; \mathbf{x}^{\prime}\right) u_{0}\left(\mathbf{x}^{\prime}\right) d \mathbf{x}^{\prime},
$$

where $G_{0}\left(\mathbf{x} ; \mathbf{x}^{\prime}\right)$ is the Greens function in the background medium and the integral is over the entire volume $V$ of the medium. For simplicity, we omit the frequency variable in (2.3) and the equations hereafter.

Eq. (2.3) is a nonlinear (Ricatti) equation. Suppose that $|\nabla \psi \cdot \nabla \psi| \ll\left|k^{2} \varepsilon\right|$, the firstorder Rytov approximation becomes

$$
\psi^{1}(\mathbf{x})=\frac{1}{u_{0}(\mathbf{x})} \int_{V} k^{2} \varepsilon\left(\mathbf{x}^{\prime}\right) G_{0}\left(\mathbf{x} ; \mathbf{x}^{\prime}\right) u_{0}\left(\mathbf{x}^{\prime}\right) d \mathbf{x}^{\prime}
$$

Note that Eq. (2.4) is only valid for small scattering angle and small velocity perturbation [22].

In literatures, to calculate the higher-order approximation of the complex phase, $\psi$ can be expanded as a series in powers of $v[4,12]$ :

$$
\psi=v \psi^{(1)}+v^{2} \psi^{(2)}+\cdots,
$$

where $v$ is a small parameter.

The higher-order Rytov series can be obtained by substituting (2.5) and (2.2) into the perturbed wave equation (2.1), and the calculation of the $n$ th-order Rytov approximation needs to solve an integral equation where the source term is composed of all the lower terms of Rytov series [4,12,27]. However, the convergence of Rytov series is still not rigorously proved, and the series was calculated up to only the third order as far as we know.

In this paper, we will present a new iterative scheme to solve the nonlinear Eq. (2.3). First, we can rewrite (2.3) as:

$$
\left\{\begin{array}{l}
p(\mathbf{x})=\int_{V}\left(\nabla \psi\left(\mathbf{x}^{\prime}\right) \cdot \nabla \psi\left(\mathbf{x}^{\prime}\right)\right) G_{0}\left(\mathbf{x} ; \mathbf{x}^{\prime}\right) u_{0}\left(\mathbf{x}^{\prime}\right) d \mathbf{x}^{\prime} \\
\psi(\mathbf{x})=\psi^{1}(\mathbf{x})+\frac{p(\mathbf{x})}{u_{0}(\mathbf{x})}
\end{array}\right.
$$

where $p(\mathbf{x})$ is an auxiliary wavefield, generated by the equivalent volume source $\nabla \psi(\mathbf{x})$. $\nabla \psi(\mathbf{x})$ which is neglected in the first-order Rytov approximation. An intuitive scheme 
for solving (2.6) is:

$$
\left\{\begin{array}{l}
p^{(n)}(\mathbf{x})=\int_{V}\left(\nabla \psi^{(n-1)}\left(\mathbf{x}^{\prime}\right) \cdot \nabla \psi^{(n-1)}\left(\mathbf{x}^{\prime}\right)\right) G_{0}\left(\mathbf{x} ; \mathbf{x}^{\prime}\right) u_{0}\left(\mathbf{x}^{\prime}\right) d \mathbf{x}^{\prime}, \\
\psi^{(n)}(\mathbf{x})=\psi^{(1)}(\mathbf{x})+\frac{p^{(n)}(\mathbf{x})}{u_{0}(\mathbf{x})}
\end{array}\right.
$$

where $\psi^{(n)}(\mathbf{x})$ is the $n$ th-order approximation to the complex phase in (2.3), and $n>1$.

Eq. (2.7) will give rise to a sequence $\psi^{(1)}(\mathbf{x}), \psi^{(2)}(\mathbf{x}), \cdots$ which is hoped to converge to the complex phase itself. However, from our numerical tests, we find that (2.7) is a computationally unstable iteration scheme, due to the numerical errors at very low and high frequency ends.

Note that the real and imaginary part of $\psi$ stand for amplitude and phase information of the scattered wavefield, respectively. Since the phase information is very important for the inversion of large-scale velocity structures, we mainly focus on improving the phase accuracy of the Rytov series using higher-order approximation. To avoid numerical instabilities and make the iterative scheme more computationally tractable, we introduce a frequency-independent traveltime perturbation field by calculating the weighted-average of the imaginary part of phase:

$$
\bar{T}(\mathbf{x})=\int W(\omega) T(\mathbf{x} ; \omega) d \omega,
$$

where $T(\mathbf{x} ; \omega)$ is a frequency-dependent traveltime perturbation field defined by

$$
T(\mathbf{x} ; \omega)=\frac{\operatorname{Im} \psi(\mathbf{x} ; \omega)}{\omega},
$$

and $W(\omega)$ is a weighting function defined by the normalized power spectrum of source wavelet spectrum $s(\omega)$

$$
W(\omega)=\frac{s(\omega) s^{*}(\omega)}{\int s(\omega) s^{*}(\omega) d \omega}
$$

The introduction of the normalized weighting function can suppress the noises arisen from the numerical calculation of the background Greens function at both low-frequency and high-frequency ends, resulting to a stable traveltime perturbation field.

Now the imaginary part of complex phase can be approximated by the product of a frequency-independent traveltime perturbation field and the angular frequency:

$$
\operatorname{Im} \psi(\mathbf{x} ; \omega) \approx \omega \bar{T}(\mathbf{x}),
$$

Substituting (2.11) into (2.7) and keeping the imaginary part of $\psi$ leads to a new iterative scheme:

$$
\left\{\begin{array}{l}
p^{(n)}(\mathbf{x})=-\int_{V} \omega^{2}\left(\nabla \bar{T}^{(n-1)}\left(\mathbf{x}^{\prime}\right) \cdot \nabla \bar{T}^{(n-1)}\left(\mathbf{x}^{\prime}\right)\right) G_{0}\left(\mathbf{x} ; \mathbf{x}^{\prime}\right) u_{0}\left(\mathbf{x}^{\prime}\right) d \mathbf{x}^{\prime} \\
\psi^{(n)}(\mathbf{x})=\psi^{(1)}(\mathbf{x})+\frac{p^{(n)}(\mathbf{x})}{u_{0}(\mathbf{x})} .
\end{array}\right.
$$


Recall that the first-order Rytov approximation also has a similar expression:

$$
\left\{\begin{array}{l}
p^{(1)}(\mathbf{x})=\int_{V} \omega^{2} \frac{\varepsilon\left(\mathbf{x}^{\prime}\right)}{c_{0}^{2}\left(\mathbf{x}^{\prime}\right)} G_{0}\left(\mathbf{x} ; \mathbf{x}^{\prime}\right) u_{0}\left(\mathbf{x}^{\prime}\right) d \mathbf{x}^{\prime} \\
\psi^{(1)}(\mathbf{x})=\frac{p^{(1)}(\mathbf{x})}{u_{0}(\mathbf{x})} .
\end{array}\right.
$$

Comparing (2.12) with (2.13), we can find that for higher-order approximation, the secondary source term in the first-order Rytov approximation is replaced with an equivalent scattering source term in the higher-order Rytov approximation, where the equivalent scattering source is given by:

$$
Q_{e}(\mathbf{x})=-\omega^{2} u_{0}(\mathbf{x})(\nabla \bar{T}(\mathbf{x}) \cdot \nabla \bar{T}(\mathbf{x})) .
$$

The direct calculation of (2.12) in the frequency domain still requires huge computational cost, due to the explicit calculation of Greens function of two arbitrary points. Therefore, by defining a time-domain equivalent scattering source $q(\mathbf{x}, t)$ :

$$
q^{(n)}(\mathbf{x}, t)= \begin{cases}-\frac{\varepsilon(\mathbf{x})}{c_{0}^{2}(\mathbf{x})} \frac{\partial^{2} u_{0}(\mathbf{x}, t)}{\partial t^{2}}, & n=1 \\ \left(\nabla \bar{T}^{(n-1)}(\mathbf{x}) \cdot \nabla \bar{T}^{(n-1)}(\mathbf{x})\right) \frac{\partial^{2} u_{0}(\mathbf{x}, t)}{\partial t^{2}}, & n>1\end{cases}
$$

the higher-order Rytov approximation can be efficiently implemented in the time domain as:

$$
\left(\frac{1}{c_{0}^{2}(\mathbf{x})} \frac{\partial^{2}}{\partial t^{2}}-\nabla^{2}\right) p^{(n)}(\mathbf{x}, t)=q^{(n)}(\mathbf{x}, t), \quad n=1,2, \cdots .
$$

Finally, the $n$ th-order Rytov approximation of the total wavefield is:

$$
u^{(n)}(\mathbf{x} ; \omega)=u_{0}(\mathbf{x} ; \omega) e^{i \operatorname{Im} \psi^{(n)}(\mathbf{x} ; \omega)} .
$$

Since only the imaginary part of the complex phase is taken to calculate the higher-order Rytov approximation, the amplitude will stay unchanged. Therefore, the neglect of the real part of the complex phase will cause amplitude differences compared with the total wavefield. As mentioned before, we only focus on improving the phase accuracy of the forward scattered wavefield for it might be benefit to future non-linear traveltime inversion in large-scale strong perturbation media.

\section{Examples}

To show the limitations of first-order Rytov approximation and the improvements of the proposed higher-order Rytov modeling method, we conducted a set of forwardscattering experiments using Gaussian-shape velocity perturbations. 


\section{Fast Gaussian perturbation}

The background velocity is a homogeneous velocity $\left(v_{0}=2 \mathrm{~km} / \mathrm{s}\right)$, and the true velocity model is a fast Gaussian anomaly embedded in a constant background. The scale of the Gaussian anomaly is $a=1.0 \mathrm{~km}$, in the form:

$$
d v(x, z)=\varepsilon v_{0} \exp \left(-\frac{r^{2}}{2 a^{2}}\right), \quad r=\sqrt{\left(x-x_{0}\right)^{2}+\left(z-z_{0}\right)^{2}},
$$

where $x_{0}=5.0 \mathrm{~km}, z_{0}=5.0 \mathrm{~km}$, and $\varepsilon$ is the percentage perturbation, $a$ is a scale parameter.

The grid number of velocity model for $x$ and $z$ directions is 1001 and 1001, respectively, and the grid interval is $0.01 \mathrm{~km}$. The experiments configuration, as shown in Fig. 1, is similar to the case of transmission tomography. The source wavelet is a Ricker wavelet with $10 \mathrm{~Hz}$ peak frequency at $(x=2.5 \mathrm{~km}, z=0 \mathrm{~km})$. A line of receivers are placed at $z=7.0$ $\mathrm{km}$, starting from $x=1.0 \mathrm{~km}$ to $9.0 \mathrm{~km}$. The wave length in the background velocity is $\lambda_{0}=0.2 \mathrm{~km}$. Therefore, the scale of anomaly is five times of wave length $\left(a=1 \mathrm{~km}=5 \lambda_{0}\right)$.

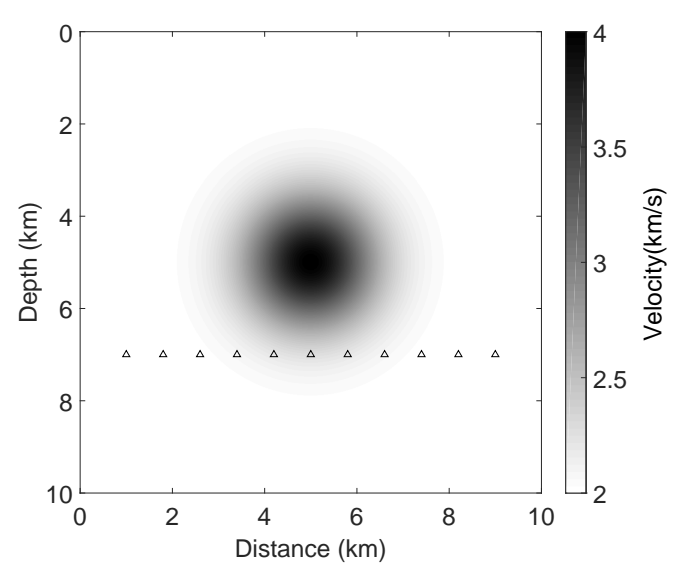

Figure 1: Velocity model with a fast Gaussian anomaly $(\varepsilon=100 \%)$ and the geometry.

To demonstrate the advantages of higher-order Rytov approximation, three models with different percentage perturbations are tested $(\varepsilon=10 \%, 50 \%, 100 \%)$. We calculated up to the fifth-order approximation and the results are shown in Fig. 2(a)-(c). In Fig. 2, the seismic waveforms simulated by the true velocity model are plotted by wiggle traces. The grey solid traveltime curve is calculated from the background model. The greendashed-, sky-blue-dashed and the red-dotted traveltime curves are calculated from the first-order, the third-order and the fifth-order Rytov approximation, respectively. Note that the traveltime is defined as the time where the maximum amplitude is detected.

In the first example ( $\varepsilon=10 \%$, Fig. 2(a)), we can see that the first-order Rytov approximation traveltime has a good agreement with the true result, and is also coincide with the third-order and the fifth-order results. The first example demonstrates that the accuracy of first-order Rytov approximation is adequate due to the small perturbation strength. 

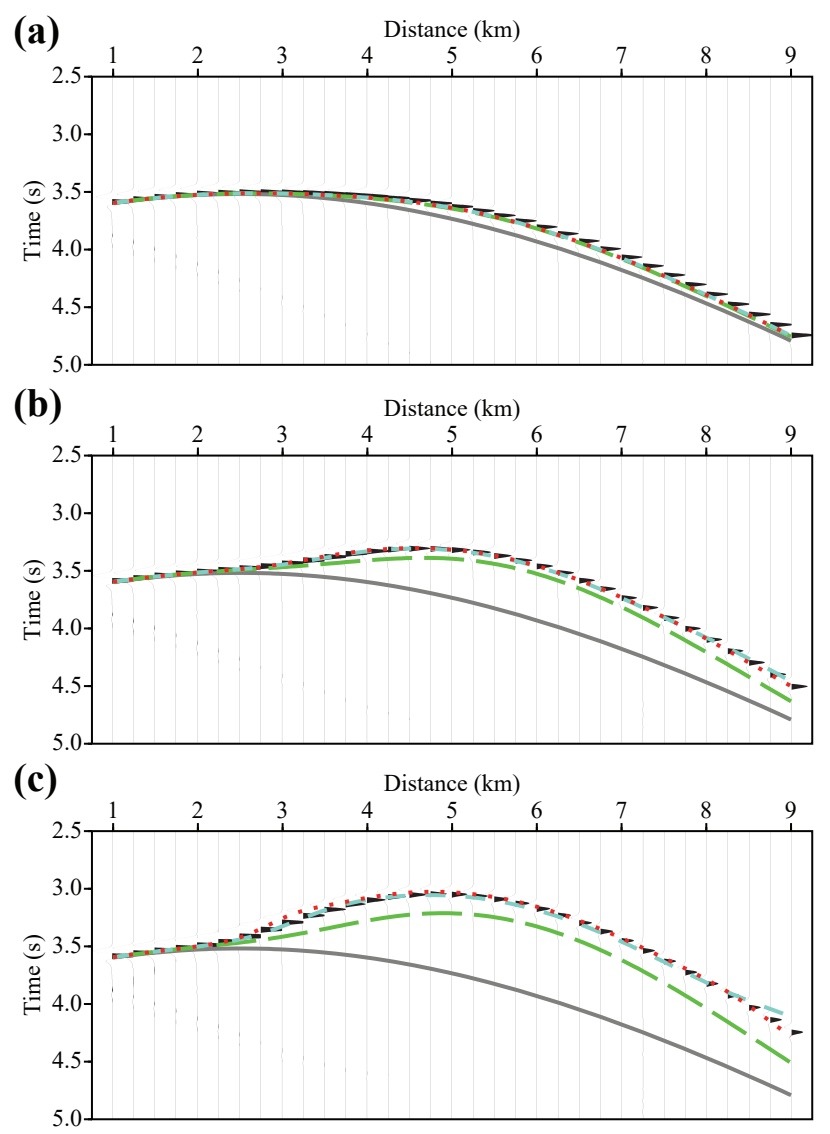

Figure 2: Traveltime comparison for the fast Gaussian ball perturbation model with (a) $\varepsilon=10 \%$, (b) $\varepsilon=$ $50 \%$, and (c) $\varepsilon=100 \%$. The background waveform is calculated from the true velocity model. The grey solid traveltime curve is calculated from the background model. The green-dashed-, sky-blue-dashed and the red-dotted traveltime curves are calculated from the first-order, the third-order and the fifth-order Rytov approximation, respectively.

In Fig. 2(b), the percentage perturbation is increased to 50\% which stands for a relatively strong perturbation, implying that the validity condition of first-order Rytov approximation is not satisfied any more. Therefore, the traveltime of first-order Rytov approximation (green-dashed curve) shows clear derivation from the waveforms in the true velocity model. On the other hand, the third-order Rytov approximation traveltime (skyblue-dashed curve) matches well with the true traveltime. In addition, the fifth-order traveltime (red-dotted curve) fits the waveforms very well, and the difference between the third-order and the fifth-order traveltime is hard to distinguish. In this case, we conclude that the third-order Rytov approximation can produce a good accuracy for traveltime prediction of forward scattering.

In Fig. 2(c), the percentage perturbation is increased to $100 \%$, and we find that the first-order traveltime dramatically deviates from the true waveforms. Nevertheless, the 
third-order traveltime is still close to the true result, except for some apparent errors at far offset range (receiver horizontal positions from $x=8500 \mathrm{~m}$ to $9000 \mathrm{~m}$ ). Furthermore, the fifth-order traveltime can match with the true waveforms everywhere, indicating that the fifth-order Rytov approximation can achieve an acceptable accuracy.

To further compare the traveltime and amplitude behaviors, Fig. 3 shows comparisons of seismic waveforms calculated by different methods for fast Gaussian perturbation models (blue curves: waveforms calculated in the true model; green curves: waveforms calculated by the first-order Rytov approximation; red curves: waveforms calculated by the fifth-order Rytov approximation; black curves: waveforms calculated by the first-order Born approximation) for $\varepsilon=10 \%$ (a), 50\% (b) and $100 \%$ (c).

From Fig. 3(a), in the area where the horizontal coordinate $x \leq 2 \mathrm{~km}$, the first-order Born, the first-order and the fifth-order Rytov modeling results are coincide with the true waveforms, because the seismic wave only penetrates the marginal part of the Gaussian velocity perturbation. As the perturbation strength increases, the seismic rays bent slightly, resulting to an amplitude focusing phenomena which can be observed in Fig. 2(b) and (c). Since the real-part of complex phase is neglected, the amplitude change cannot be recovered, and we can see the amplitude differences in Fig. 3(b) and (c). For $x \geq 4$ $\mathrm{km}$, the first-order Born modeling results only show strong amplitudes but no traveltime shifts although the perturbation strength is small. Wu and Zheng [28] demonstrated that for large-scaled perturbation, the first-order Born approximation cannot get satisfied result. On the contrary, the proposed higher-order Rytov approximation results, especially the fifth-order result, can predict the traveltime shifts very well.

From Fig. 2(b), the traveltime shifts between the true and background waveforms become obvious for $x \geq 3 \mathrm{~km}$. Due to the large perturbation strength, the first-order Rytov approximation is not valid any more. Therefore, in Fig. 3(b), the green-dashed curves (first-order Rytov approximation) have apparent traveltime deviation from the true waveforms, but the fifth-order Rytov approximation (red-dotted curves) still match the true waveforms very well.

For the $\varepsilon=100 \%$ example, as shown in Fig. 3(c), the traveltime deviations between the first-order Rytov approximation result and the true waveform become more evident. But the fifth-order Rytov approximation results only have small traveltime shifts compared with the dominant wave period, except that the amplitude cannot be recovered due to the neglect of the real part of the complex phase. Since the defocusing effect of the positive Gaussian anomaly will cause an amplitude decrease, the amplitudes of the total wavefields are weaker than the background wavefield. However, the Born modeling results, in both Fig. 3(b) and (c), show very strong amplitude (about 1 order of magnitude higher than the true waveforms).

\section{Slow Gaussian perturbation}

Next, we will test the higher-order Rytov approximation in the presence of low velocity anomaly. The shape Gaussian anomaly is identical to the previous perturbation described 
(a)

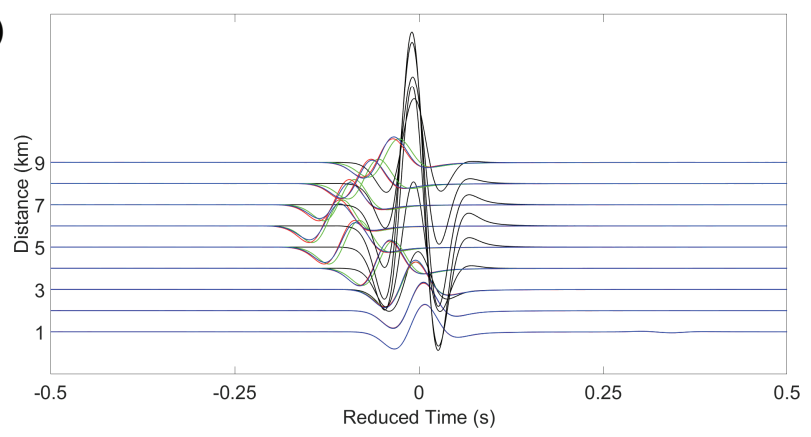

(b)

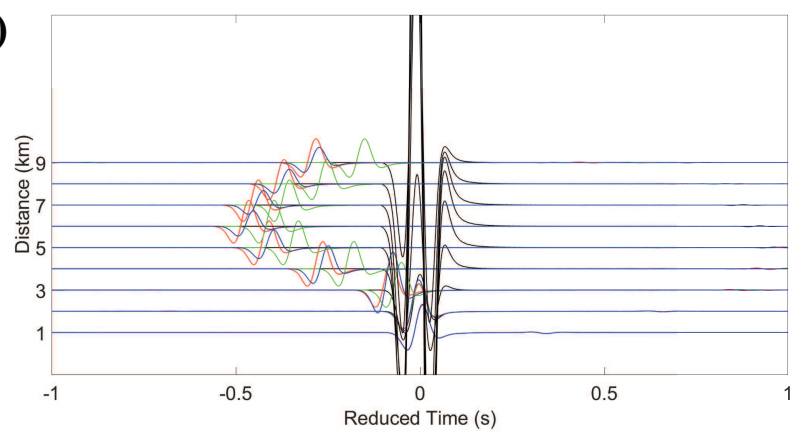

(c)

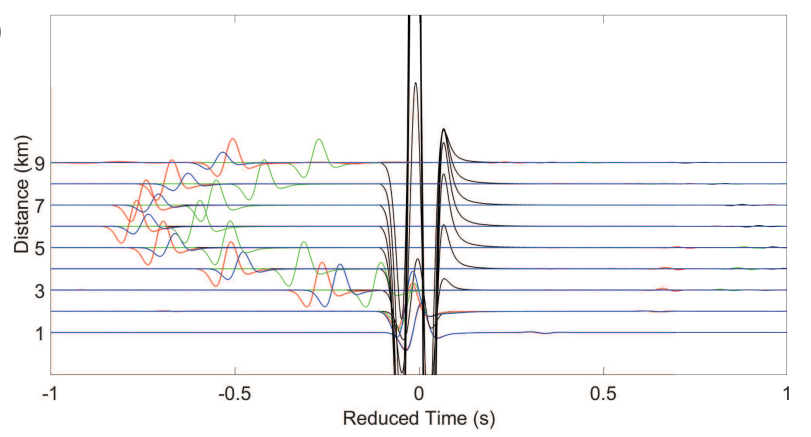

Figure 3: Comparison of seismic waveforms for fast Gaussian perturbation models: (a) $\varepsilon=10 \%$, (b) $\varepsilon=50 \%$, and (c) $\varepsilon=100 \%$. The blue curves are waveforms in true model; the green and red curves stand for the first-order and the fifth-order Rytov modeling results, respectively; the black curves are the first-order Born modeling results. The reduced traveltime is defined as the true traveltime minus the background traveltime.

in (3.1), except that now the percentage perturbation are negative values: $\varepsilon=-10 \%$, $-25 \%,-50 \%$. Besides, we increase the homogeneous background velocity to $3 \mathrm{~km} / \mathrm{s}$ $\left(v_{0}=3 \mathrm{~km} / \mathrm{s}\right)$ to reduce numerical dispersion of the finite-difference algorithm. In all examples, the Rytov series is also calculated to the fifth-order.

In the second example $(\varepsilon=-10 \%$, Fig. $4(\mathrm{a}))$, the results are similar to the positive perturbation case in Fig. 2(a). Both the first-order and higher-order traveltime have a good agreement with the true result. When the percentage perturbation is increased to 

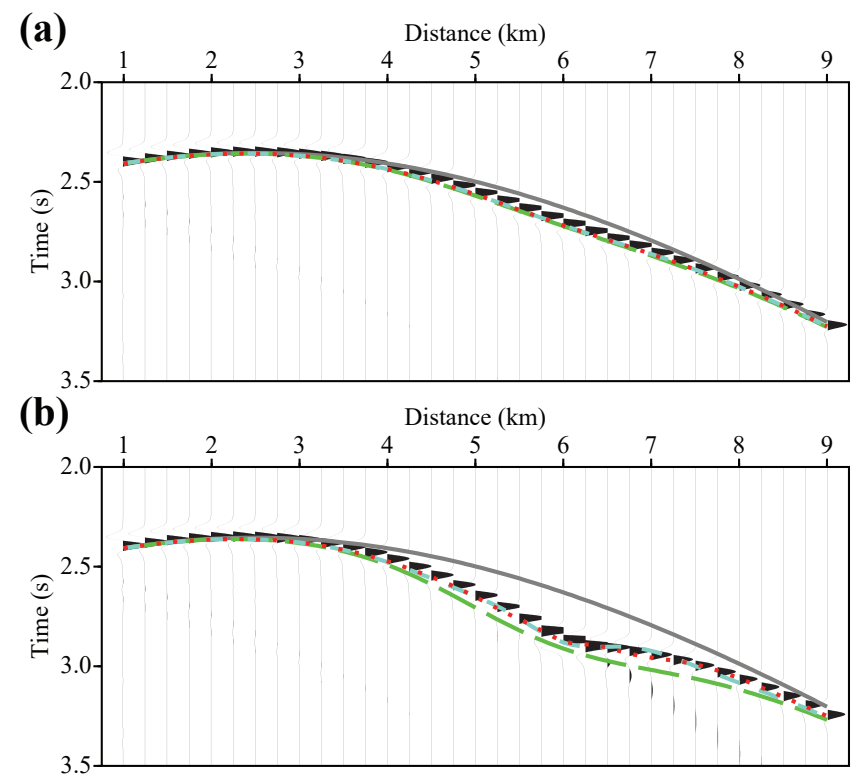

Figure 4: Traveltime comparison for the slow Gaussian ball perturbation model with (a) $\varepsilon=-10 \%$ and (b) $\varepsilon=-25 \%$. The background waveform is calculated from the true velocity model. The grey solid traveltime curve is calculated from the background model. The green-dashed-, sky-blue-dashed and the red-dotted traveltime curves are calculated from the first-order, the third-order and the fifth-order Rytov approximation, respectively.

$-25 \%$, as shown in Fig. 4(b), the first-order Rytov approximation result (green-dashed curve) has large traveltime errors for $x=5-8 \mathrm{~km}$, due to the accumulated phase errors neglected in the first-order approximation. On the contrary, the third-order traveltime (sky-blue-dashed curve) has a big improvement over the first-order approximation. Furthermore, the fifth-order result (red-dotted curve) has a better agreement with the true record. Finally, the percentage perturbation is increased to $-50 \%$. However, the multiarrivals phenomena are observed due to the presence of strong slow-velocity Gaussian anomaly. In such case, it is hard to calculate the gradient of complex phase accurately, resulting to a divergent Rytov series. Further research is needed to calculate an accurate gradient in the presence overlapping wavefronts and multi-arrivals.

We can also make a comparison for the traveltime and amplitude behaviors. Fig. 5 shows comparisons of seismic waveforms calculated using the negative Gaussian perturbation models with $\varepsilon=-10 \%$ (a) and $-25 \%$ (b). For the small perturbation case $(\varepsilon=-10 \%$, Fig. 5(a)), in the area where $x \geq 2 \mathrm{~km}$, both the Born and Rytov modeling results are close to with the true waveforms. For $x \leq 5 \mathrm{~km}$, the first-order Rytov approximation traveltime (the green curves) gradually deviates from the true result, but the fifth-order approximation result still matches with the true result very well. When the strength is increased to $-25 \%$, the waveform mismatches are more apparent. From Fig. 5(b), for $x=4-9 \mathrm{~km}$, the first-order Rytov approximation results have a large traveltime difference compared with the true result. But the traveltime errors for fifth-order result are still very small 
(a)

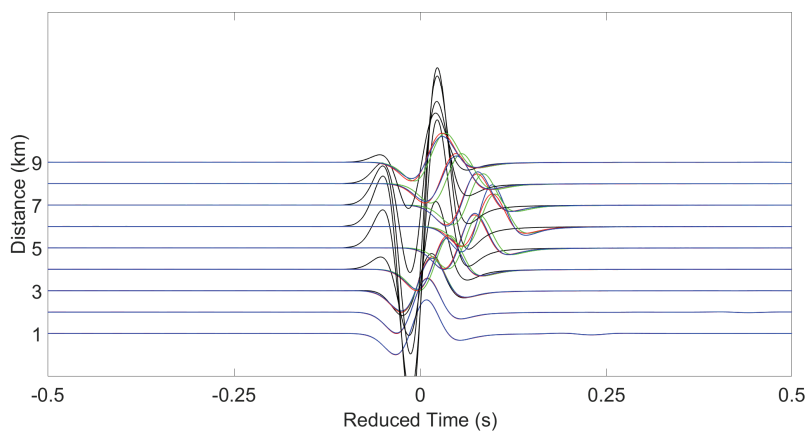

(b)

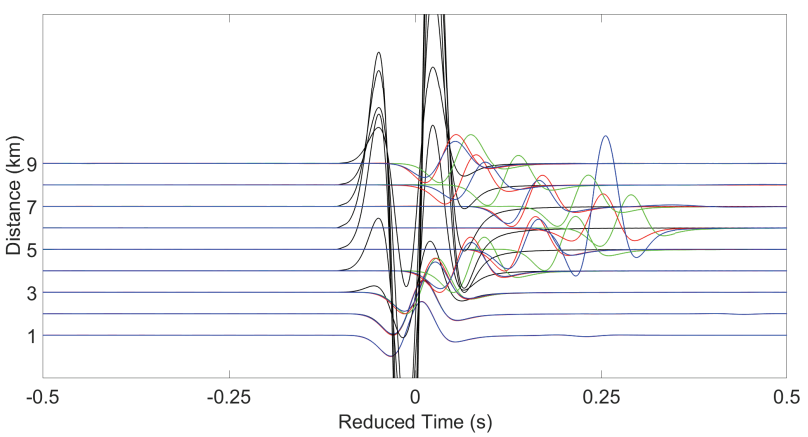

Figure 5: Comparison of seismic waveforms for slow Gaussian perturbation models: (a) $\varepsilon=-10 \%$ and (b) $\varepsilon=-25 \%$. The blue curves are waveforms in true model; the green and red curves stand for the first-order and the fifth-order Rytov modeling results, respectively; the black curves are the first-order Born modeling results. The reduced traveltime is defined as the true traveltime minus the background traveltime.

compared with the dominant wave period. Due to the focusing effect of the low-velocity Gaussian anomaly, the seismic amplitude of the total wavefield at $x=6 \mathrm{~km}$ is amplified dramatically. As previously discussed, the amplitude change of the total wavefield cannot be recovered by the higher-order Rytov modeling method. However, our main objective is to recover the phase information which is hoped to be used for future nonlinear traveltime inversion.

\section{Discussions and conclusion}

To approximate the complex phase from lower to higher order gradually, we introduced an equivalent scattering source composed of the product of incident wavefield and gradient-square of the $n$ th-order traveltime perturbation field. Since we mainly focus on the phase accuracy of the complex phase, the imaginary part of complex phase is then approximated using the weighted-average of a traveltime perturbation field multiplied by frequency. The physical interpretation for the phase-traveltime variable change is that only primary waves are considered. Therefore, in the presence of multi-arrivals, 
the equivalent scattering source should be handled carefully due to the multi-directions of the wavefield.

The neglect of the real part of the complex phase implies that the amplitude cannot be recovered accurately. Nevertheless, considering that the phase information plays an important rule for the subsequent non-linear traveltime inversion, these assumptions are still reasonable and acceptable.

In this paper, we developed an equivalent source method which can update the imaginary part of the complex phase iteratively, and the higher-order scattered wavefield can be approximated by multiplying the incident wavefield with the exponent of the imaginary part of the complex phase. Numerical examples demonstrated that the accuracy of the first-order Rytov approximation is not enough if the perturbation strength is strong, while the proposed method can produce an improved accuracy for traveltime (phase) prediction. Even if the percentage perturbation is 100 per cent, the fifth-order Rytov approximation can produce a satisfying result. The application of the proposed higherorder Rytov approximation method to non-linear traveltime inversion is our future research.

\section{Acknowledgments}

This work is supported by WTOPI (Wavelet Transform On Propagation and Imaging for seismic exploration) Research Consortium and WPI (Wave phenomena and inversion imaging) Research Group. We are grateful to our Consortium sponsors for their financial support and allowing us to publish our research results. WPIs research are financially supported by National Natural Science Foundation of China (41604091, 41704111, 41774126), the great and special project (2016ZX05024-001, 2016ZX05006-002).

\section{References}

[1] Wu, R.S. and Aki, K. Elastic wave scattering by a random medium and the small-scale inhomogenetities in the lithosphere. J. Geophys. Res., 90, 10261-10273, 1985.

[2] Wu, R.S. The perturbation method in elastic wave scattering. Pure and Appl. Geophys., 131, 605-637, 1989.

[3] Chernov, L. A. Wave Propagation in a Random Medium. McGraw-Hill, New York, 1960.

[4] Tatarskii, V. L. The Effects of the Turbulent Atmosphere on Wave Propagation, (translated from Russian) National Technical Information Service, 1971.

[5] Ishimaru, A. Wave Propagation and Scattering in Random Media, vol. II, Academic Press, New York, 1978.

[6] Aki, K. Scattering of P Waves under the Montana Lasa. J. Geophys. Res. 78, 13341346, 1973.

[7] Flatte, S. M. and Wu, R.S. Small-scale structure in the lithosphere and asthenosphere deduced from arrival-time and amplitude fluctuations at NORSAR. J. Geophys. Res., 93, $66016614,1988$.

[8] Wu, R.S. and Flatt, S.M. Transmission Fluctuations across an Array and Heterogeneities in the Crust and Upper Mantle. Pure Appl. Geophys., 132, 175196, 1990. 
[9] Devaney, A. J. A Filtered Back Propagation Algorithm for Diffraction Tomography. Ultrasonic Img., 4, 336350, 1982.

[10] Devaney, A. J. Geophysical Diffraction Tomography. Trans. IEEE GE-22, 313, 1984.

[11] Wu, R.S. and Toks?z M. N. Diffraction Tomography and Multisource Holography Applied to Seismic Imaging. Geophysics, 52, 1125, 1987.

[12] Tsihrintzis, G. A. and Devaney A. J. Higher-order (nonlinear) diffraction tomography: Reconstruction algorithms and computer simulation. IEEE Trans. Image Processing, 9(9), 1560$1572,2000$.

[13] Huang, L.J., Fehler, M., Roberts, P., and Burch, C. C. Extended Local Rytov Fourier Migration Method. Geophysics, 64, 15351545, 1999.

[14] Woodward, M. J. Wave-equation tomography. Geophysics, 57, 15-26, 1992.

[15] Dahlen, F.A., Nolet, G., and Huang, S.-H. Frchet kernels for finite-frequency traneltime-I Theory. Geophys. J. Int., 141, 157-174, 2000.

[16] Xie, X.B., and Yang, H. The finite-frequency sensitivity kernel for migration residual moveout and its applications in migration velocity analysis. Geophysics, 73, S241-S249, 2008.

[17] Xu, W., Xie, X.B., Geng, J. Validity of the Rytov Approximation in the Form of FiniteFrequency Sensitivity Kernels. Pure and Applied Geophysics, 172(6), 14091427, 2015.

[18] DeWolf, D. A. Validity of Rytovs Approximation. J. Opt. Soc. Amer., 57(8), 1057-1058, 1967.

[19] Brown, W.P. Validity of the Rytov Approximation in Optical Propagation Calculations. J. Opt. Soc. Amer., 56(8), 1045-1052, 1966.

[20] Brown, W.P. Validity of the Rytov Approximation. J. Opt. Soc. Amer., 57(12), 1539-1542, 1967.

[21] Keller, J. B. Accuracy and validity of the Born and Rytov approximation. J. Opt. Soc. Amer., 59, 1003-1004, 1969.

[22] $\mathrm{Wu}, \mathrm{R} . S$. Wave propagation, scattering and imaging using dual-domain one-way and onereturn propagators. Pure and Appl. Geophys., 160(3/4), 509-539, 2003.

[23] Tsihrintzis, G. A. and Devaney A. J. Higher Order (Nonlinear) Diffraction Tomography. Inversion of the Rytov Series. IEEE Transactions on information theory, 46 (5), 1748-1761, 2000.

[24] Manning, R. M. Beam wave propagation with the second Rytov perturbation approximation. Radiophysics and Quantum Electronics, 39(4), 287-295, 1996.

[25] Kim, B.C., and Tinin, M. V. The second-order Rytov approximation and residual error in dual-frequency satellite navigation systems. Waves in Random and Complex Media, 19(2), 284-304, 2009.

[26] Flatte, S. M. Sound Transmission through a Fluctuating Ocean. Cambridge University Press, 1979.

[27] Yura, H.T., Sung, C.C., Clifford, S.F. and Hill, R.J. Second-order Rytov approximation. J. Opt. Soc. Amer., 73(4), 500-502, 1983.

[28] Wu, R.S, and Zheng Y. Non-linear partial derivative and its De Wolf approximation for nonlinear seismic inversion. Geophysical Journal International, 196(3), 1827-1843, 2014. 